Chirurg 2019 90 (Suppl 2):S79 https://doi.org/10.1007/s00104-019-0855-y Online publiziert: 13. Februar 2019

(c) Springer Medizin Verlag GmbH, ein Teil von Springer Nature 2019

\section{Originalpublikation}

Acharya A, Markar SR, Sodergren MH et al. (2017) Meta-analysis of adjuvant therapy following curative surgery for periampullary adenocarcinoma. Br J Surg 104:814-822

Hintergrund. Periampulläre Karzinome sind eine seltene und heterogene $\mathrm{Tu}$ morentität. Bei fehlenden Fernmetastasen stellt die chirurgische Resektion die Therapie der Wahl dar. Während beim Pankreaskarzinom die adjuvante Therapie ein etablierter Therapiebestandteil ist, dessen Benefit durch mehrere Arbeiten belegt wurde, ist die Datenlage beim periampullären Karzinom weniger eindeutig. Die hier vorgestellte Metaanalyse fasst die Ergebnisse aktueller Studien zur adjuvanten Therapie beim periampullären Karzinom zusammen.

Methoden. Studien zur adjuvanten Therapie nach kurativ intendierter Resektion von periampullären Karzinomen in englischer Sprache aus den Jahren 2000 bis 2015 wurden eingeschlossen. Voraussetzung war eine Nachbeobachtungszeit von mindestens 3 Jahren. Die Analyse erfolgte anhand eines Random-EffectsModells.

Ergebnisse. Die Einschlusskriterien für die Metaanalyse erfüllten 14 Studien mit einer Gesamtzahl von 1671 Patienten (904 in der Kontrollgruppe und 767 mit adjuvanter Therapie). Darunter waren 6 randomisiert kontrollierte Studien

Dieser Beitrag wurde erstpubliziert in Der Chirurg (2017) 88:889-889. https://doi.org/10. 1007/s00104-017-0509-x

M. Schrempf $\cdot$ M. Anthuber

Klinik für Allgemein-, Viszeral- Transplantationschirurgie, Klinikum Augsburg, Augsburg, Deutschland

\title{
Metaanalyse zur adjuvanten Therapie des periampullären Adenokarzinoms
}

(RCT), 2 prospektive Kohortenstudien und 6 retrospektive Studien. Die adjuvante Therapie bestand in 4 der Studien aus einer alleinigen Chemotherapie und in 8 Studien aus einer Radiochemotherapie. Das mediane 5-Jahres-Überleben betrug $37,5 \%$ in der Kontrollgruppe und $40,0 \%$ in der Gruppe mit adjuvanter Therapie und unterschied sich damit nicht signifikant (Hazard Ratio [HR] $=1,08 ; 95 \%$-Konfidenzintervall [KI] 0,91-1,28; $p=0,067)$. Die Analyse der 6 RCT ergab ebenfalls keinen Unterschied im 5-Jahres-Überleben (HR = 1,01; $95 \%$-KI 0,80-1,26; $p=0,102$ ). Angaben zu Nebenwirkungen der adjuvanten Therapie waren in 9 der 14 Studien verfügbar. Schwere (WHO-Grad 3) oder lebensbedrohliche (WHO-Grad 4) Nebenwirkungen erlitten 247 (32,2\%) der 767 Patienten mit adjuvanter Therapie.

\section{Diskussion und Fazit des Reviewers.}

Diese Metaanalyse konnte keinen Überlebensvorteil durch eine adjuvante Therapie bei periampullären Karzinom nachweisen. Allerdings erlitt knapp ein Drittel aller Patienten unter adjuvanter Therapie schwere Nebenwirkungen. Dies dürfte die tatsächliche Häufigkeit von Nebenwirkungen noch unterschätzen, da 5 Studien keine Toxizitätsangaben enthielten. Im Gegensatz zu Lymphknotenstatus und Differenzierungsgrad zeigte in dieser Analyse nur ein fortgeschrittenes T-Stadium eine Assoziation mit einem kürzeren Langzeitüberleben. Die Frage, ob Patienten im Stadium T3 oder T4 von einer adjuvanten Therapie profitieren, wurde jedoch aufgrund der zu geringen Fallzahl nicht beantwortet. Ein weiterer Schwachpunkt der Metaanalyse ist die
Heterogenität der einzelnen Studien in Bezug auf Therapieschemata, Dosierung der Substanzen und Anzahl der Zyklen.

Periampulläre Karzinome können immunhistologisch vereinfacht in einen intestinalen und einen pankreatikobiliären Typ eingeteilt werden. Der pankreatikobiliäre Subtyp ist mit einem schlechteren Langzeitüberleben assoziiert und zeigt ein besseres Ansprechen auf Gemcitabinbasierte Therapien als der intestinale Subtyp [1]. Solange jedoch nicht durch weitere Arbeiten geklärt wird, ob bestimmte klinische und histologische Subtypen tatsächlich von einer adjuvanten Therapie profitieren, kann keine generelle Empfehlung zur adjuvanten Therapie beim periampullären Karzinom gegeben werden.

\section{Korrespondenzadresse}

Prof. Dr. M. Anthuber

Klinik für Allgemein-, Viszeral- Transplantationschirurgie, Klinikum Augsburg Stenglinstr. 2, 86156 Augsburg, Deutschland matthias.anthuber@klinikum-augsburg.de

Interessenkonflikt. M. Schrempf und M. Anthuber geben an, dass kein Interessenkonflikt besteht.

\section{Literatur}

1. Schiergens TS, Reu S, Neumann J et al (2015) Histomorphologic and molecular phenotypes predict gemcitabine response and overall survival in adenocarcinoma of the ampulla of Vater. Surgery 158:151-161 